\title{
Features of relativistic electron radiation in storage rings
}

\author{
Oleg Shishanin ${ }^{1, *}$ \\ ${ }^{1}$ Moscow State University of Food Production, Volokolamskoe highway, 11, 125080 Moscow, Russia
}

\begin{abstract}
The formulation of the problem is due to the fact that the spectral properties of radiation in alternating magnetic fields, as compared with homogeneous fields, differ little, but the angular characteristics depend significantly on betatron oscillations. Because the dynamics of an electron in the magnetic systems of accelerators is rather complicated, a number of simplifications has been made here. The asymptotic formulas for the spectral and angular distributions of the radiation intensity with the first quantum correction have been obtained by Schwinger's operator method. For them, as well as for the angular characteristics of synchrotron light, approximate expressions are given, allowing researchers to determine the desired properties of photon emission at certain points of the electron orbit.
\end{abstract}

\section{Introduction}

The existing theory of synchrotron radiation has heretofore been developed mainly for uniform magnetic fields [1-4]. In [5, 6], the question of the influence of betatron oscillations on radiation has not been specifically considered. In actuality, electrons accelerating in periodic magnetic fields perform transverse oscillations which can noticeably affect the angular and polarization properties of the emitted radiation. In consequence of this, it would be reasonable to formulate the photon emission problem in the non-uniform magnetic field. Such an analysis would, among other things, motivate the development of more comprehensive beam diagnostics and enhance the precision of experiments involving polarized synchrotron radiation.

The effect of betatron oscillations on synchrotron radiation was first discussed in [7] for an axisymmetric magnetic field. Then, we carried out an analysis of the properties of the radiation emitted in the FD, FODO, FOFDOD systems, etc. [8, 9]. In the present paper, using the beta function concept, we attempt to extend the theory to more general magnetic structures, including storage rings.

\section{Betatron function method}

According to present views, the small vertical motion of charged particles in a circular accelerator can be described by the following function:

$$
z=\sqrt{\frac{\beta_{z} A_{z}}{\pi}} \cos \left(\int \frac{d s}{\beta_{z}}+\delta_{0}\right),
$$

\footnotetext{
*e-mail: olegsh55@gmail.com
} 
where $A_{z}$ is the emittance, $\beta_{z}$ is the beta function which depends on the orbital length $s$, and $\delta_{0}$ is the initial phase.

Let $\varphi=s / R_{0}$ be a generalized azimuthal angle, where $R_{0}$ is the mean radius (viz., the total orbit length divided by $2 \pi$ ) in magnetic systems with straight sections.

In cylindrical co-ordinates, the velocity components are given by

$$
v_{x}=\dot{\rho} \cos \varphi-\left(R_{0}+\rho\right) \sin \varphi \dot{\varphi}, v_{y}=\dot{\rho} \sin \varphi+\left(R_{0}+\rho\right) \cos \varphi \dot{\varphi}, v_{z}=\dot{z},
$$

where the dot denotes the total derivative with respect to t.

When $\rho$ is added to $R_{0}$, the direction of a narrow cone of radiation varies moderately. Thus the radial oscillations influence the angular radiation distributions only slightly and we may assume that the particle moves on an average circular orbit of radius $R_{0}$.

The expression for the angular velocity assumes the form:

$$
\dot{\varphi}=\frac{\omega_{0}}{1+k}\left[1-\frac{\rho}{R_{0}}+\frac{3}{2} \frac{\rho^{2}}{R_{0}^{2}}+\frac{1}{R^{2}} \int(z \dot{z}-\rho \dot{\rho}) f(\varphi) d t\right] .
$$

\section{Angular and spectral properties of radiation}

Now we employ the operator method [2,7] for studying the properties of the emitted synchrotron light. We will suppose that the radiation vector $\vec{\kappa}=\omega \vec{n} / c$, where $\vec{n}=\{0 ; \sin \theta, \cos \theta\}$ lies in the YZ plane, and $\theta$ is the spherical angle.

We define the linear polarization vectors in the wave zone as follows:

$$
\vec{e}_{\sigma}=\{1,0,0\}, \quad \vec{e}_{\pi}=\{0, \cos \theta,-\sin \theta\}
$$

Here, the first vector corresponds to the electric field component lying in the orbital plane and the second one is orthogonal to this plane. In the first quantum approximation the components of the emission intensity can be written as

$$
\begin{gathered}
\frac{d W_{\sigma}}{d^{3} \kappa}=\frac{c e^{2}}{(2 \pi)^{3} R_{0}} \frac{v^{\prime}}{v}\left|\int d t v_{x} \exp \left(i \frac{v^{\prime}}{v}(\omega t-\vec{\kappa} \vec{r})\right)\right|^{2}, \\
\frac{d W_{\pi}}{d^{3} \kappa}=\frac{c e^{2}}{(2 \pi)^{3} R_{0}} \frac{v^{\prime}}{v}\left|\int d t\left(v_{y} \cos \theta-v_{z} \sin \theta\right) \exp \left(i \frac{v^{\prime}}{v}(\omega t-\vec{\kappa} \vec{r})\right)\right|^{2},
\end{gathered}
$$

where $\omega=v \omega_{0} /(1+k)$ and $v^{\prime}=v(1+h \omega / E)$.

The angle $\varphi$ can be reckoned from any point of the orbit. Then, the expansion parameters are given by $\varphi \sim m_{0} c^{2} / E, \tau=N \varphi(N$ is the number of magnetic periods in the accelerator or storage ring), $\cos \theta(\theta \sim \pi / 2), \rho / R_{0}$, and $z / R_{0}$. Using these, we also take $\sin \varphi \sim \varphi-$ $\varphi^{3} / 6, \sin \theta \sim 1$ in the parameter

$$
\Phi=\omega t-\vec{\kappa} \vec{r}=\frac{v \omega_{0}}{1+k}\left[t-\frac{1}{c}\left(R_{0}+\rho\right) \sin \varphi \sin \theta-\frac{z}{c} \cos \theta\right] .
$$

To determine $\varphi$, it is necessary to carry out the integration. In particular, in the zeroth approximation $\varphi=\omega_{0} t /(1+k)$.

From the equality

$$
v^{2}=\dot{\rho}^{2}+\dot{z}^{2}+R^{2} \omega_{0}^{2}\left[1+\frac{\rho^{2}}{R_{0}^{2}}+\frac{2}{R^{2}} \int(z \dot{z}-\rho \dot{\rho}) f(\varphi) d t\right]
$$


the quantity $R \omega_{0} / c$ can be solved.

The velocity components are

$$
\frac{v_{x}}{c} \approx \frac{\dot{\rho}}{c}-\varphi, \quad \frac{v_{y}}{c} \approx \beta, \quad v_{z}=\dot{z} .
$$

We now introduce a new variable $u=-v_{x} / c=\varphi-\dot{\rho} / c$ and carry out expansions of the form:

$$
\begin{gathered}
z=\left.z\right|_{\tau=0}+\left.\frac{d z}{d \tau}\right|_{\tau=0} \cdot \tau+\ldots \approx z_{0}+\frac{v_{z}}{c} R_{0} \varphi \\
\frac{\omega_{0}}{1+k} \int d t\left[\int(z \dot{z}-\rho \dot{\rho}) f(\varphi) d t\right] \approx \text { const }+\frac{1}{N} \tau \int d t(z \dot{z}-\rho \dot{\rho}) f(\varphi)
\end{gathered}
$$

and so on.

Retaining terms up to the third order, we obtain

$$
\Phi=\left[1-\beta \sin \theta+\frac{u^{2}}{6}-\frac{v_{z}}{c} \cos \theta+\frac{1}{2}\left(\frac{v_{z}}{c}\right)^{2}\right] u+\text { const } .
$$

In the ultrarelativistic case we have $1-\beta \sin \theta \approx \varepsilon / 2$, where $\varepsilon=1-\beta^{2} \sin ^{2} \theta$.

Integrating Eqs.(3) and averaging over the initial phases we get for the spectral-angular distributions

$$
\begin{gathered}
\frac{d W_{\sigma}(v)}{d \Omega}=\frac{c e^{2} v v^{\prime}}{12 \pi^{4} R_{0}^{2}} \int_{0}^{2 \pi} d \delta \varepsilon_{1}^{2} K_{2 / 3}^{2}\left(\frac{v^{\prime}}{3} \varepsilon_{1}^{3 / 2}\right), \\
\frac{d W_{\pi}(v)}{d \Omega}=\frac{c e^{2} v v^{\prime}}{12 \pi^{4} R_{0}^{2}} \int_{0}^{2 \pi} d \delta \varepsilon_{1} \varepsilon_{2} K_{1 / 3}^{2}\left(\frac{v^{\prime}}{3} \varepsilon_{1}^{3 / 2}\right),
\end{gathered}
$$

where

$$
\begin{gathered}
\varepsilon_{1}=1-\beta^{2}+\varepsilon_{2}, \quad \varepsilon_{2}=\left(\cos \theta-\left.\frac{v_{z}}{c}\right|_{\tau=0}\right)^{2}, \\
\left.\frac{v_{z}}{c}\right|_{\tau=0}=\alpha \cos \delta, \quad \alpha=\left.\sqrt{\frac{A_{z}}{\pi}}\left[\frac{1}{\sqrt{\beta_{z}}} \sqrt{1+\left(\frac{1}{2} \frac{d \beta_{z}}{d s}\right)^{2}}\right]\right|_{\tau=0} .
\end{gathered}
$$

Formulas (4) can mainly be used for storage rings. For their practical application we must bear in mind that the graph of the beta function is usually known for a closed trajectory. Consequently, we can define its value at the point where photon emission is recorded, while the derivative can be approximated by the ratio $\Delta \beta_{z} / \triangle s$.

For bending magnets the graph of the beta function is almost linear; in this case we can use the slope of the linear segment to approximate the derivative.

Introducing the angle $\psi$ reckoned from the orbital plane, we can approximate $\cos \theta$ by $\psi$.

Let us now discuss the method of calculating the integrals in (4). Initially, we may go over to the Airy function

$$
K_{1 / 3}\left(\frac{2}{3} x^{3 / 2}\right)=\pi \sqrt{\frac{3}{x}} A i(x), \quad K_{2 / 3}\left(\frac{2}{3} x^{3 / 2}\right)=-\frac{\pi \sqrt{3}}{x} A i^{\prime}(x)
$$

with $x=(v / 2)^{2 / 3} \varepsilon$ and employ well-known integral tables [10]. 
In the case of greatest interest (for small amplitudes of the oscillations or small crosssections of the bunch) one can use an expansion in terms of the parameter $q^{2}=\alpha^{2} / 2 \varepsilon$. Then, in the classical approximation we obtain the following expressions:

$$
\begin{gathered}
\frac{d W_{\sigma}(v)}{d \Omega}=W\left\{\left(A i^{\prime}\right)^{2}+2 x^{2} q^{2}\left[2 x g U+(1+2 g) A i A i^{\prime}\right]+\frac{1}{2} x^{2} q^{4}\left[12 x^{2} g(1+g) A i^{2}+\right.\right. \\
\left.\left.x\left(3+24 g+16 x^{3} g^{2}\right) U+3\left(1+16 x^{3} g+24 x^{3} g^{2}\right) A i A i^{\prime}\right]\right\}, \\
\frac{d W_{\pi}(v)}{d \Omega}=W x\left\{\left(g+q^{2}\right) A i^{2}+2 x g q^{2}\left(2 x g U+5 A i A i^{\prime}\right)+3 x q^{4} A i A i^{\prime}+\right. \\
\left.\frac{1}{2} x^{2} g q^{4}\left[\left(39+16 x^{3} g^{2}\right) U+28 x g A i^{2}+8 x^{2} g(14+3 g) A i A i^{\prime}\right]\right\},
\end{gathered}
$$

where

$$
\begin{gathered}
W=\frac{2^{1 / 3} c e^{2} v^{2 / 3}}{\pi^{2} R_{0}^{2}}, \quad g=\psi^{2} / \varepsilon, \quad U=x A i^{2}+\left(A i^{\prime}\right)^{2}, \\
\varepsilon=1-\beta^{2}+\cos ^{2} \theta=\frac{1}{\gamma^{2}}\left(1+\gamma^{2} \psi^{2}\right) .
\end{gathered}
$$

We note that the parameter $x$ is the argument both of the function $A i$ and its derivative.

Assuming $q^{2}=0$ (there are no vertical oscillations) and replacing $R_{0}$ by $R$, these formulae can be transformed into expressions for the uniform magnetic field.

In the neighbourhood of the orbital plane $x$ is a suitable expansion parameter. Here we use the Airy function $V(x)=\sqrt{\pi} A i(x)$ with the initial Fock conditions: $V(0)=0.629271, V^{\prime}(0)=$ -0.458745 [11]. This function, along with its derivative, can be expanded in convergent power series. In this case, the braces in (9) must be replaced by, respectively,

$$
\begin{gathered}
V^{\prime}(0)^{2}\left\{1+\frac{1}{3} x^{3}\left[2+3 q^{2}\left(2+3 q^{2}\right)(1+4 g)+5 q^{6}\right]\right\}+ \\
V(0) V^{\prime}(0) x^{2}\left(1+2 q^{2}+4 q^{2} g+\frac{3}{2} q^{4}\right)
\end{gathered}
$$

and

$$
\begin{gathered}
V^{2}(0)\left(g+q^{2}\right)+V(0) V^{\prime}(0) x\left(2 q^{2}+3 q^{4}+2 g+10 g q^{2}\right)+ \\
\frac{1}{2} V^{\prime}(0)^{2} x^{2}\left[2 q^{2}+6 q^{4}+5 q^{6}+g\left(2+20 q^{2}+39 q^{4}\right)+8 q^{2} g^{2}\right]
\end{gathered}
$$

From these formulae we can observe that at $\theta=\pi / 2$ the $\sigma$-component is less than the same component for a uniform magnetic field, and the magnitude of the $\pi$-component is not equal to zero.

Peaks of the curves plotted in accordance with formulae (4) will generally be below the graphs that are built to the uniform magnetic field at the same energy $E$ and bending radius $R$. In the plane of the equilibrium orbit the radiation will not be completely linearly polarized.

We will first discuss the spectral properties of the emission. If in (5) we retain $\cos \theta$ instead of $\psi$ and integrate with respect to the spherical angle $\theta$, we derive spectral formulae almost coinciding with the analogous expressions for a uniform magnetic field. There are corrections of the $\alpha^{2} / R_{0}^{2}$ order and similar terms to the radial vibrations.

Summing expressions (4) over the entire spectrum, we can obtain angular characteristics. If the betatron oscillations are small $(p<<1)$ we get simple formulae:

$$
\frac{d W_{\sigma}}{d \Omega}=\frac{7 W_{1}}{\varepsilon^{5 / 2}}\left(1-\frac{5}{4} p+\frac{35}{4} p g\right),
$$




$$
\frac{d W_{\pi}}{d \Omega}=\frac{5 W_{1}}{\varepsilon^{5 / 2}}\left(g+\frac{1}{2} p-\frac{35}{4} p g+\frac{63}{4} p g^{2}\right),
$$

where $W_{1}=c e^{2} / 32 \pi R_{0}^{2}$. Setting $p=0$ and replacing parameter $R_{0}$ with $R$ in $W_{1}$ we regain the usual expressions for uniform magnetic field. This part of the paper refers to the critical wavelength of the synchrotron light.

\section{Conclusion}

Special experiments were carried out on this topic for a long time on the electron synchrotrons with different energies [3]. In general, we can note a good agreement of these works with the given theory. All authors emphasize the important role of the axial electron oscillations in the formation of the spectral-angular properties.

Basic formulae (4) determine the properties of the radiation for modern accelerators. Here the parameters $\cos \theta$ or $\psi$, which are often used in literature, add in $\varepsilon_{2}$ the scalar term, formed by a variable vector of the particle velocity. That is why the radiative scattering increases.

What can these expressions give at large amplitudes of oscillations? At higher oscillation amplitudes the $\pi$-component exhibits a peak instead of the minimum at $\theta=\pi / 2$. Furthermore, it is expected that the $\sigma$-component will attain a small local minimum in the orbital plane for the extremal amplitudes and, in addition, the $\pi$-component will generate symmetric local hollows.

Thus, based on our theoretical study we propose to search for these effects in experiments on existing machines with a higher degree of accuracy than previously. We are talking only about the storage rings. Moreover, these tests have been carried out at fixed wavelengths and, apparently, these experiments are recommended to perform for the total angular distributions.

Since the considered synchrotron radiation, is incoherent at wavelengths which are of the greatest practical interest our results can be applied to the electron beams. Specifically, the cross-section of a typical bunch from which the radiation is emitted constitutes an ensemble of charged particles with various amplitudes. Recognizing this, we can re-interpret the amplitude parameter $B$ in our formulae as a mean squared value using the following arguments: the longitudinal beam distribution can be taken into account specifying the injector scatter of electrons and carrying out averaging over initial phases.

\section{References}

[1] J. Schwinger, Phys. Rev. 75, 1912 (1949)

[2] J. Schwinger, Proc. Nat. Acad. Sci. 40, 132 (1954)

[3] A.A. Sokolov and I.M. Ternov, Synchrotron Radiation (Akademie Verlag, Berlin, 1968)

[4] A.A. Sokolov and I.M. Ternov, Radiation from Relativistic Electron (AIP, New York, 1986)

[5] H. Wiedemann, Synchrotron Radiation (Springer-Verlag, 2003)

[6] A. Hofmann, The Physics of Synchrotron Radiation (Cambrigde University Press, 2004)

[7] V.Ch. Zhukovsky and O.E. Shishanin, Zh. Exp. Teor. Phys. 34, 729 (1972)

[8] O.E. Shishanin, Zh. Exp. Teor. Phys. 76, 547 (1993)

[9] O.E. Shishanin, Nucl. Instr. and Meth. A. 558, 74 (2006)

[10] M. Abramowitz and A. Stegun, Handbook of Mathematical Functions (Dover, 1970)

[11] G.D. Jakovleva, Tables of Airy Functions and Their Derivatives (in Russian) (Nauka, Moscow, 1969) 